Teknokultura. Revista de Cultura Digital y Movimientos Sociales

ISSNe: $1549-2230$

http://dx.doi.org/10.5209/TEKN.73983

\title{
Repensar la robótica y la inteligencia artificial desde la ética de los cuidados ${ }^{1}$
}

\author{
Núria Vallès-Peris ${ }^{2}$
}

Recibido: 3 de febrero de 2021 / Aceptado: 1 de abril de 2021 Open peer reviews

Resumen. En este artículo propongo la utilización de la ética de los cuidados como marco teórico y político para repensar la 'robótica' y la 'inteligencia artificial' (IA) en el ámbito de la salud. Utilizando la perspectiva teórica de los Estudios de Ciencia y Tecnología, desarrollo una aproximación a partir de la idea de continuum entre cuidados, política, ética y tecnologías. Propongo una reflexión sobre las controversias en torno a la robótica y la IA desde un posicionamiento no ingenuo, desde una lógica de conflicto y confrontación con los imaginarios que acompañan el mercado de la innovación tecnológica. Conceptualmente esta propuesta gira en torno a dos nociones: la heterogeneidad y la creatividad, nociones que expresan la potencia radical de la ética de los cuidados como utopía que desafía la utopía neoliberal. A partir de estas consideraciones articulo una manera de comprender las relaciones entre los humanos y los robots que busca superar la tradicional relación diádica humano-máquina. Tomando como unidad de análisis el entramado de relaciones de cuidados de las que participa el robot (Robot Embedded in a Network -REN-), emergen nuevas controversias que permiten visibilizar las prácticas cotidianas de cuidados con estos artefactos, y las relaciones de desigualdad que las acompañan. Del mismo modo, para poder garantizar un diseño e introducción de tecnologías responsables, al servicio del bien común y del bienestar individual y colectivo, apunto a la necesidad de integrar en el debate a los diversos actores que participan en los cuidados, así como también de establecer mecanismos de vigilancia y escrutinio público permanente en el diseño e introducción de robots en el ámbito de la salud.

Palabras clave: Estudios de Ciencia y Tecnología; imaginarios; interacción humano-robot; robots de cuidado; tecnología responsable.

\section{[en] Rethinking robotics and artificial intelligence from the ethics of care}

\begin{abstract}
In this paper I propose the use of the ethics of care as a theoretical and political framework for rethinking 'robotics' and 'artificial intelligence' (AI) in healthcare. Using the theoretical perspective of Science and Technology Studies, I develop an approach based on the idea of a continuum between care, politics, ethics and technologies. I propose a reflection on the controversies surrounding robotics and AI from a non-naïve position, from a logic of conflict and confrontation with the imaginaries that accompany the technological innovation market. Conceptually, this proposal revolves around two notions: heterogeneity and creativity, and how they express the radical potential of the ethics of care as a utopia that challenges neoliberal utopia. From these considerations I articulate a way of understanding human-robot relations that seeks to overcome the traditional dyadic human-machine relationship. Taking as a unit of analysis the network of care relations in which the robot is embedded (Robot Embedded in a Network -REN-), new controversies emerge that make visible the daily practices of care with these artefacts, and the relations of inequality that accompany them. Similarly, in order to guarantee the design and introduction of responsible technologies, at the service of the common good and individual and collective well-being, I suggest the need to integrate the various actors involved in care into the debate, as well as to establish mechanisms for monitoring and permanent public scrutiny of the design and introduction of robots in healthcare.
\end{abstract}

Keywords: care robots; human-robot interaction; imaginaries; responsible technology; Science and Technology Studies.

Sumario. 1. Introducción. 2. Los robots de cuidados. 3. La ética de los cuidados en las tecnologías de la salud. 4. La responsabilidad de la robótica y la IA de los cuidados. 5. Conclusiones. 6. Referencias.

Cómo citar: Vallès-Peris, N. (2021). Repensar la robótica y la inteligencia artificial desde la ética de los cuidados. Teknokultura. Revista de Cultura Digital y Movimientos Sociales, 18(2), 137-146. http://dx.doi.org/10.5209/TEKN.73983

\footnotetext{
Fundación "la Caixa” [LCF/PR/RC17/10110004].

2 Universitat Autònoma de Barcelona/Barcelona Science and Technology Studies Group (STS-b) (España)

E-mail: nuria.valles@uab.cat; https://orcid.org/0000-0003-4150-761X
} 


\section{Introducción}

Durante las últimas décadas los robots y la 'inteligencia artificial' (IA) se han ido introduciendo progresivamente en hospitales y otros entornos de salud. Juntamente con la biotecnología, la nanotecnología o las tecnologías relacionadas con la neurociencia, la robótica y la IA se presentan como grandes promesas de la medicina y los cuidados. Con la COVID-19 las expectativas y desarrollo alrededor de estas tecnologías ha recibido un nuevo impulso, por su capacidad de ser utilizadas para reducir los contactos físicos entre personas y evitar de ese modo el contagio y propagación del virus (Kritikos, 2020). Esta situación ha reforzado dinámicas ya existentes, agudizando la necesidad de articular un debate público alrededor de sus efectos y las numerosas controversias que generan. A pesar de que la proliferación de los robots ha supuesto también una creciente reflexión académica acerca de sus implicaciones éticas, representada por el nacimiento de la 'roboética' (Veruggio, 2005), el desarrollo de la industria robótica no ha ido acompañado en la misma medida de un debate público acerca de su potencial impacto en nuestras vidas y nuestras relaciones (FRA, 2020). Las discusiones existentes, además, se enmarcan en un contexto de creciente incertidumbre, en el que el poder de la tecnocracia, la lógica especulativa y los imaginarios utópicos y distópicos, aceleran y superan la capacidad de anticipar sus consecuencias y mesurar críticamente su influencia.

El propósito de este artículo es indagar en la ética de los cuidados como uno de los marcos teóricos y políticos desde los que repensar y discutir sobre la robótica y la IA en el ámbito de la salud. Esta propuesta se dibuja a partir de la conjunción de la ética de los cuidados con los Estudios de Ciencia y Tecnología (STS en su acrónimo en inglés). Este mosaico configura un planteamiento desde la idea de continuum entre cuidados, política, ética y tecnología, una propuesta que parte de una visión crítica del desarrollo y debate ético dominante, y subraya la necesidad de articular nuevos imaginarios alrededor de la robótica y la IA.

Recogiendo el aliento de resistencia y esperanza de propuestas como la ética nómada de Braidotti (2000) o la ética especulativa de Puig de la Bellacasa (2011), entiendo la ética de los cuidados como una aproximación privilegiada para construir nuevos marcos de análisis y discusión de la tecnociencia en general, y de la robótica y la IA en los cuidados en particular; una ética disruptiva con las perspectivas dominantes alrededor de las implicaciones sociales y éticas del desarrollo tecnológico en la salud, que respire algo así como un optimismo de combate del que hablaba Serres (2013); una aproximación a los debates éticos y sociales que contribuya a crear imaginarios de futuro con las tecnologías, al servicio del bien común y la buena vida individual y colectiva, integrando criterios de justicia y equidad.

Para desarrollar esta propuesta he organizado el artículo alrededor de tres apartados. En el primer apartado explico cómo me acerco a estos artefactos desde los STS. Seguidamente indago en las nociones de heterogeneidad y creación, como ideas fundamentales sobre las que construir una aproximación desde la ética de los cuidados. Finalmente, desarrollo una serie de consideraciones que emergen desde la aproximación propuesta.

\section{Los robots de cuidado}

Actualmente, lo que se conoce como la 'nueva robótica' propone desarrollar robots para interactuar y colaborar con personas en ambientes no controlados (Schaal, 2007). A menudo como pruebas piloto, como parte de investigaciones o proyectos experimentales, los robots son introducidos progresivamente en hospitales u otros entornos sanitarios, especialmente dirigidos a niñas y niños, personas mayores o personas con algún tipo de discapacidad (Mejia y Kajikawa, 2017).

\subsection{Los imaginarios y las condiciones de posibilidad de la robótica}

El desarrollo de este tipo de artefactos, conocidos genéricamente como robots sociales (Breazeal, 2011), está íntimamente relacionado con la integración de múltiples avances tecnológicos, especialmente con el desarrollo de la IA y las tecnologías de Colaboración entre Robots y Humanos (HRC) (Vallès-Peris y Domènech, 2020a). La HRC y la IA permiten interacciones seguras entre los robots y los humanos, una fácil programación y ajuste a las necesidades particulares, y hacen posible que los robots funcionen como sistemas de información móviles e interactivos en hospitales, residencias de ancianos y/o otros entornos sanitarios. Sin embargo, tal y como proponen los estudios constructivistas de las tecnologías (Bijker, 2009), un artefacto no es un mero objeto con una serie de funcionalidades, sino que encierra toda una red de relaciones en la que las consideraciones técnicas, científicas, políticas, económicas o éticas están íntimamente vinculadas en un todo (Callon, 1998).

El surgimiento de cualquier artefacto tiene que ver con los diversos sesgos, valores o relaciones de poder existentes durante su proceso de creación y diseño, condiciones sociohistóricas que se inscriben en ese artefacto (Castoriadis, 2005). De este modo, la creación y diseño de los robots de cuidado no puede desvincularse de la servidumbre al capitalismo neoliberal en el que vivimos, del poco valor social y político que se da a los cuidados, ni de la división sexual del trabajo que organiza los cuidados de forma desigual entre mujeres y hombres (Tronto, 2018).

Los periódicos y los estudios académicos informan periódicamente sobre la falta de enfermeras, profesorado, personal cuidador y servicio doméstico, cuestión que se agrava con el hecho de que la población vive más años que antes. Es lo que se conoce como la 'crisis de los cuidados'. En el modelo neoliberal la solución a la crisis de los cuidados se articula desde el mercado: frente a la falta de estado supuestamente defendida por este modelo, es el mercado quien se erige para cubrir las necesidades humanas (Tronto, 2018). En este contexto el crecimiento de la robótica en el ámbito de la salud 
no puede desvincularse de su potente impacto económico en el mercado de la innovación tecnológica. Según la International Federation of Robotics (IFR), el nuevo sector en expansión son los robots de servicios, entre los que se incluyen los robots médicos (categoría que integra, entre otros, los robots de cuidados). En 2017 se vendieron un total de 109.000 unidades de robots de servicios y la previsión era pasar a las 736.000 unidades en 2021. Para los robots médicos, se esperaba pasar de 1.900.000 millones de dólares facturados en 2017 a 9.600.00 millones en 2021 (IFR, 2018).

A pesar de que la crisis de los cuidados es un argumento utilizada a menudo para explicar la necesidad de desarrollar la robótica de los cuidados, la cuestión de los cuidados prácticamente no aparece en los debates a su alrededor a excepción de algunas valiosas propuestas (van Wynsberghe, 2013). Es paradigmático en este sentido que en las diferentes guías y mecanismos regulatorios de los sistemas de IA, que incluyen los robots, no se contemplen las tensiones que pueden surgir entre los intereses comerciales o de negocio y cuestiones vinculadas con el bienestar individual y colectivo o las necesidades y organización de los cuidados (Hagendorff, 2020).

Otro relato o supuesto dominante en torno a la robótica de los cuidados está directamente relacionado con el imaginario del 'agente racional' (Vallès-Peris y Domènech, 2020a), aquel que actúa para lograr el mejor resultado en caso de incertidumbre (Russell y Norvig, 2003). Para alcanzar este objetivo se utilizan diferentes modelos computacionales, como la clásica manipulación de símbolos o la IA basada en el conocimiento y el aprendizaje automático o la IA basada en datos (Steels y Mantaras, 2018). Estos modelos construyen una agencia en función de una comprensión específica del éxito que se mueve entre dos polos: la fidelidad a la forma en que los humanos actúan, en un extremo, y lo que se considera el ideal de inteligencia y racionalidad, en el otro. Sin embargo, esta cualidad de 'mejor solución' atribuida a la robótica y la IA presenta algunos problemas.

Por un lado, diversas investigaciones alertan de la amplia gama de técnicas de gestión de datos utilizadas en aplicaciones maliciosas, prácticas comerciales o problemas sociales en los que no interviene en absoluto una IA adecuada (Pistono y Yampolskiy, 2016). Esta tensión entre el imaginario del agente racional como la mejor solución y sus usos fraudulentos, es la fuente de muchas directrices, normativas y recomendaciones sobre los riesgos que acompañan estas tecnologías, donde predominan cuestiones como la vigilancia y la privacidad, la manipulación del comportamiento, la opacidad de los sistemas de IA o los sesgos de los sistemas de decisión (Muller, 2020).

Por otro lado, aunque es innegable que la robótica y la IA han tenido un desarrollo exponencial, existe una brecha importante entre las preocupaciones éticas en torno a sus supuestas funcionalidades y las posibilidades reales actuales (Hagendorff, 2020) development and application of artificial intelligence (AI). Ideas como una IA fuerte o la conciencia de las máquinas, por ejemplo, son consideradas como absurdas por expertos técnicos (Calo, 2017). Más allá de los robots bailarines de Boston Dynamics o de la androide Call de Alien: Resurrection, actualmente el desarrollo de robots humanoides, autónomos, inteligentes y conscientes parece ser más una historia de ciencia ficción que de la realidad (Steels y Mantaras, 2018).

\subsection{La negociación del código técnico y la potencia disruptiva de los cuidados}

Los imaginarios acerca de la robótica son el producto de un contexto sociohistórico específico. Pero según Castoriadis (1975) la creación es siempre ex-nihilo, es decir, consiste en hacer surgir nuevas formas de ser que no existían, que no pueden ser explicadas suficientemente por las condiciones históricas precedentes. De este modo cualquier elemento social se compone de dos polos irreductibles que no pueden existir el uno sin el otro: el imaginario instituido, que es el resultado de un proceso sociohistórico; y el imaginario instituyente o imaginario radical, a partir del cual se realizan nuevas creaciones (Castoriadis, 1975). No se trataría solamente de contemplar la configuración de un artefacto como un proceso determinado por su contexto. Como recoge Feenberg (2010) en su tesis de la instrumentalización, los artefactos no se explican solamente por las condiciones de posibilidad de realización de su contexto sociohistórico de diseño y producción. Cuando los robots de cuidado o cualquier otro dispositivo tecnológico se introducen en el mundo de la vida cotidiana, lo que son y las mediaciones que posibilitan dependen de su uso en un determinado entorno. En ese entorno se configuran las condiciones de posibilidad de utilización, que pueden ser diferentes de las que se pretendía originalmente y de las que estaban contempladas en su diseño (Feenberg, 2010).

La tesis de instrumentalización debe ser vista como una respuesta indeterminada al determinismo tecnológico: el diseño de una tecnología no determina la sociedad ni las mediaciones que configura, porque esas mediaciones están conformadas por factores tanto del artefacto como de su entorno de uso (Coeckelbergh, 2012). La idea que subyace a esta tesis es que las tecnologías emergen como el resultado contingente de luchas y negociaciones, configurándose el artefacto y su código técnico a partir de las relaciones. Esta noción es esencial para la propuesta que planteo alrededor de los robots, permitiéndome vincular la aproximación constructivista y la ética de las tecnologías con la ética de los cuidados. ¿Por qué? Porque las relaciones de cuidados implican formas de relación que van más allá de la lógica de poder y dominación de los mercados de innovación tecnológica $\mathrm{y}$, por la tanto, conforman dinámicas de conflicto y negociación en los procesos de los que emergen y en los que se utilizan las nuevas tecnologías en el ámbito de la salud.

\section{La ética de los cuidados en las tecnologías de la salud}

Desde la idea de continuum entre tecnologías y cuidados, en los siguientes párrafos desarrollo una aproxima- 
ción a los cuidados a partir de las nociones de heterogeneidad y creatividad: la heterogeneidad entraña la noción de (co)creación entre los cuidados, los robots y las relaciones de las que estos participan; y la creatividad hace referencia a la capacidad de los cuidados de confrontar el debate ético dominante en robótica, centrado en funcionalidades y características técnicas.

\subsection{La heterogeneidad de los cuidados}

A pesar de que las experiencias y las prácticas de cuidar pueden ser identificadas, investigadas y entendidas concreta y empíricamente, 'los cuidados' son ambivalentes en su significado y ontología (Puig de la Bellacasa, 2017). Los cuidados se han explorado desde la economía (Carrasco, 2019), la psicología (Martínez et al., 2011), la enfermería (Domínguez Alcón, 2018), la antropología (Duclos y Criado, 2020), en estudios sobre diseño (Sánchez Criado y Rodríguez Giralt, 2017), sobre procesos migratorios (Agrela Romero, Martín Palomo, y Langa Rosado, 2010), desde el activismo (Precarias a la deriva, 2004) o desde muchas otras aproximaciones; la lista sería inacabable. Sin embargo, a partir de estos diversos estudios y ámbitos de conocimiento, seguramente no sería posible (ni deseable) establecer un criterio unívoco común para explicar qué son los cuidados (Puig de la Bellacasa, 2017).

Asumiendo esta complejidad y diversidad en la comprensión de los cuidados, es especialmente sugerente la reconocida definición de Fisher y Tronto (1990):

El cuidado es todo lo que hacemos para mantener, continuar y reparar nuestro mundo para que podamos vivir en él lo mejor posible. Este mundo incluye nuestros cuerpos, nosotras mismas y nuestro entorno, todo lo cual tratamos de entrelazar en una compleja red que sostiene la vida (Fisher y Tronto, 1990, p. 40).

El interés de esta manera de comprender los cuidados no recae en una descripción exhaustiva ni en una definición categórica específica sobre qué son los cuidados. Lo que hace la aproximación de Fisher y Tronto (1990) tan atractiva, y a la vez tan radical, es su manera heterogénea de comprender los cuidados, inseparables de las consideraciones económicas, políticas, simbólicas o materiales que los conforman. «El cuidado es todo lo que hacemos», incluye las prácticas de cuidados, lo que habitualmente se considera el trabajo doméstico; los afectos y el sentido emocional que implican los cuidados; y la organización y concepción política que supone gestionar y regular todo aquello que sostiene las relaciones de cuidados. «[T] odo lo que hacemos para mantener, continuar y reparar nuestro mundo» desde sus dimensiones de trabajo, afectos y política. Cuidar como una relación personal y a la vez un entramado colectivo, que «incluye nuestros cuerpos, nosotros mismos y nuestro entorno", porque a pesar de que los cuidados son una cuestión humana, no atañe solamente a los humanos, sino a todo nuestro mundo, con sus ballenas, sus líneas de metro, sus dientes de león, sus ríos y sus tacitas de café, «entrelazándonos en una red compleja de vida sos- tenible», de vidas interconectadas e interdependientes. Esto no implica que la dependencia sea un valor absoluto en todas las situaciones -como muestran las luchas de los movimientos para la vida independiente (MoyàKöhler, 2018), ni tampoco que los cuidados tengan que ser una obligación moral en todas las decisiones, situaciones o prácticas (el poder disruptivo de elegir no cuidar de muchas mujeres que se suponía que tenían que cuidar). Es una de las maneras de mirar las relaciones, no la única (Puig de la Bellacasa, 2017).

Desde la ética de los cuidados analizar las relaciones de cuidados implica rechazar la lógica según la cual hay un conocimiento o un bien previo y verdadero sobre cómo se debe cuidar. Desde los estudios STS que analizan la utilización de tecnologías en el ámbito de la salud, esta lógica se ha recogido en la noción de 'ética empírica de los cuidados'. La ética empírica gira entorno a la idea de que los cuidados están localizados en las prácticas de las personas que, con la ayuda de procesos, protocolos, rutinas o máquinas, actúan para conseguir el buen cuidar (Willems y Pols, 2010). Una idea clave que subyace a este planteamiento es la capacidad mediadora de los artefactos tecnológicos. Las tecnologías posibilitan determinadas relaciones de los humanos con el mundo que de otro modo no habrían sido posibles. Sin embargo, las tecnologías no son intermediarias neutrales, sino mediadoras activas que contribuyen a la formación de las percepciones y la interpretación que los humanos hacemos de la realidad: son moral materializada, ofrecen propuestas materiales sobre cómo actuar (Verbeek, 2008).

Esta idea supone que el debate ético no recae en grandes temas filosóficos, sino en aspectos vinculados con el día a día. De este modo, en el ámbito de la robótica el interés no recaería solamente en cuestiones como si es adecuada la sustitución de humanos por robots (Sharkey, 2008), si es deseable establecer vínculos afectivos con las máquinas (Sharkey y Sharkey, 2012; Sparrow y Sparrow, 2006), o la responsabilidad en caso de daño o perjuicio (Matsuzaki y Lindemann, 2016), sino que también se fijaría en identificar las prácticas, miedos y esperanzas que configuran las relaciones de cuidados cuando se introducen robots en contextos y situaciones específicas. Por ejemplo, cómo en un hospital pediátrico una enfermera de planta consigue que los niños y niñas de las diferentes habitaciones salgan al pasillo para alimentar al robot-mascota que lleva con ella; o cómo se ha decidido en una reunión que a partir de ese momento en las terapias para autismo se utilizarán unos robots que ha regalado una gran empresa de robótica.

Consecuentemente, los resultados de este tipo de aproximación no pueden ser soluciones prescriptivas a problemas genéricos. Por ejemplo, no responden a preguntas del tipo ¿hay que poner o no un robot con webcam para poder monitorizar durante todo momento los pacientes que están en cuidados intensivos?, sino que ofrece propuestas sugestivas en relación con un problema específico. Siguiendo con el ejemplo ¿cuáles pueden ser los argumentos a favor y en contra de poner una webcam?, ¿cuál es el posicionamiento de familiares, el paciente, el personal médico, el departamento de innovación, etc.?, o ¿cómo podemos retocar el robot parar 
que encaje en las rutinas, prácticas, necesidades y preocupaciones en ese contexto? (Vallès-Peris y Domènech, 2020b).

\subsection{La capacidad creativa de los cuidados}

La cuestión primordial sobre la que argumenta la ética de los cuidados es que: para que las vidas interdependientes sean posibles en el mundo en que vivimos, tiene que haber algunas formas de cuidado realizándose en algún lugar de este mundo, que posibiliten vivir en él (Puig de la Bellacasa, 2017). Esta idea de los cuidados como elemento común de nuestras vidas y de las relaciones que establecemos entre nosotros y nuestro entorno, es lo que posibilita comprender la ética de los cuidados como una propuesta política, necesaria para pensar las tecnologías que nos rodean.

Si nos tomamos el cuidado en serio como punto de partida de nuestras teorías sociales y políticas, este ofrece una alternativa al paradigma del fundamentalismo de mercado que impera actualmente. A menudo se critica el cuidado por ser una forma utópica de enfocar la vida social y política. Pero hoy quiero girar las tornas: lo verdaderamente utópico es el fundamentalismo de mercado, y si empezamos a entenderlo de esta forma, entonces podremos empezar a explorar nuevas posibilidades de cambio político (Tronto, 2018, p. 8).

Para desarrollar este argumento Tronto (2018) utiliza las tesis de Polany. Según Polany (2015), a pesar de que las economías neoliberales presumen de que el sistema económico está controlado, regulado y dirigido por los precios del mercado, este tipo de funcionamiento revierte el orden normal de las sociedades humanas. Lo que en realidad encontramos es que, en el supuesto libre mercado, las regulaciones y el mercado han crecido juntos. La idea de que el mercado se autorregula requeriría de la separación entre los ámbitos económico y político, erigiéndose el mercado como ente independiente. Sin embargo, desde una perspectiva sociológica heterogénea, el sistema económico no puede separarse del resto de funciones de las sociedades humanas, el mercado no puede existir separado de la sociedad.

Que el mercado fuera el único director del destino de la sociedad y su entorno resultaría en la destrucción de la sociedad misma, porque los humanos y el mundo en el que viven no se pueden 'utilizar' indiscriminadamente. La sociedad desechada y tiranizada al servicio del mercado se convertiría en accesoria del sistema económico y éste acabaría destruyéndolo todo (Polany, 2015). Como resultado, esta sociedad de mercado nunca ha existido y se podría considerar un ideal utópico (Block y Somers, 1984). La utopía (o 'distopía') de una sociedad en la que los humanos no necesitarían amarse ni cuidarse para vivir juntos, en la que la indiferencia y el repliegue sobre uno mismo fueran los únicos garantes de las sociedades humanas, tiene resonancias demasiado inquietantes (Dupuy, 1991). Consecuentemente, la economía de mercado genera constantemente mecanismos políticos y sociales que limitan su lógica, para poder asegurar la supervivencia.
El movimiento del molino satánico, como Polany (2015) se refería a esa acción desregulada y su lógica, permite comprender el modelo neoliberal como utópico. Frente a esa utopía, el deseo de una sociedad y unas formas de desarrollo tecnológico responsables es también una fuerte utopía que puede hacer sentir su voz.

Si llegamos a entender el neoliberalismo como una utopía, si vemos el neoliberalismo como un freno para conseguir una sociedad más responsable, y si presentamos la idea de una sociedad más responsable como una alternativa viable, entonces es posible desafiar su poder ideológico (Tronto, 2018, p. 9).

El desafío ideológico de la ética de los cuidados se sustenta en la idea de que las personas, en lugar de ser criaturas de mercado, son criaturas que viven en relaciones de cuidado mutuo. No es accesorio en mi propuesta acercarme al neoliberalismo y a la ética de los cuidados como utopías. El desarrollo tecnológico de la robótica y la IA es indudable, pero también lo es su lógica tecnocrática y el crecimiento de mercado generado. Esta cuestión no puede desvincularse del debate ético a su alrededor, que en gran parte discute sobre controversias de un hipotético escenario futuro, en el que los más visionarios son los que piden consideraciones éticas y sociales y dan la errónea impresión de que los debates están abordando situaciones reales y no hipotéticas (van der Plas, Smits y Wehrmann, 2010). La discusión ética alrededor de los robots es muy futurista y parece que la investigación sobre los aspectos éticos y sociales se está llevando a cabo con la intención de anticipar objeciones y reticencias (Nordmann y Rip, 2009). La prospectiva sobre la utilización de este tipo de artefactos opera en la reflexión ética más como una idea quimérica, que como una tecnología concreta (Šabanović, 2010). Y el debate transcurre en una tensión entre imaginarios utópicos y distópicos (van der Plas et al., 2010), un fenómeno relativamente frecuente en las nuevas tecnologías, lo que algunos autores llaman la ética especulativa de los desarrollos tecnológicos (Grunwald, 2010).

A pesar de la creciente mercantilización de las tareas de cuidados o de la instrumentalización de la etiqueta 'responsable' y 'cuidado', los procesos de (co) contrucción entre las tecnologías y los cuidados son una realidad tan tozuda como los mercados (López Gómez, 2015; Winance, 2006). Los imaginarios, las utopías y los debates éticos a su alrededor forman parte de la red de relaciones heterogéneas que conforman los artefactos (Vallès-Peris, Argudo-Portal y Domènech, en prensa), las prácticas o los cuidados. Este tipo de discusiones no son mera sublimación o evasión, sino que posibilitan la creatividad, la emergencia de cosas que sobrepasan la realidad establecida. Esta capacidad de creación está condicionada por las posibilidades del contexto sociohistórico, a la vez que supera la realidad instituida, y genera una matriz genérica de sentido, esperanza y transformación social (Carretero Pasín, 2005). La ética de los cuidados planteada como utopía no solamente propone un mundo ideal y una alternativa al capitalismo neoliberal, sino que se convierte también en movilizadora, impregna la realidad sociotécnica y la renueva. 


\section{La responsabilidad de la robótica y la IA en los cuidados}

Un marco de discusión desde la ética de los cuidados permite complejizar los debates existentes alrededor de los riesgos y funcionalidades de la robótica y la IA, posibilitando la emergencia de nuevos temas. Las nociones de heterogeneidad y creatividad desarrolladas desde los STS son clave en esta propuesta. Pero no es suficiente hablar de ensamblajes heterogéneos y de la capacidad de resistencia y creatividad en la apropiación de las tecnologías, porque en esos ensamblajes no todos los agentes que participan son iguales ni participan de la misma manera. Hablar de la robótica y la IA desde lo ético-político de la ética de los cuidados es comprometerse con aquellos y aquellas que difícilmente podrían hacer oír su voz sobre lo que les concierne (Puig de la Bellacasa, 2011), identificar qué imaginarios y preocupaciones prevalecen en el diseño tecnológico, qué relaciones de poder materializan los artefactos y a qué mundo contribuyen.

Desde este marco de discusión emergen nuevas consideraciones sobre las implicaciones sociales y éticas de los robots de cuidado, a la vez que se esbozan nuevos imaginarios que acompañen estos artefactos. Estas consideraciones hacen referencia a: (1) comprender los dispositivos robóticos como materialidad relacional, como artefactos que participan en entramados; (2) lo cual permite visibilizar una serie de prácticas cotidianas de cuidados con robots y; (3) al mismo tiempo, proponer una serie de transformaciones en el entramado de relaciones en el que se integra ese robot, incorporando procesos de seguimiento y debate constante con todos los actores.

\subsection{Los dispositivos robóticos como materialidad relacional. Robot Embedded in a Network}

Los robots ejecutan una serie de tareas específicas en los entornos de atención de la salud (como facilitar la relación de los pacientes con espacios de fuera del hospital o disminuir su angustia en el preoperatorio) o modifican las formas de ejecutar determinadas acciones del personal sanitario (como medir las constantes, hacer intervenciones minuciosas, etc.). Cuando un robot se introduce en la red particular de relaciones de un hospital, el robot y las relaciones de cuidados se (co)transforman: cambia la manera en que entendemos y practicamos los procesos de atención y cuidados, del mismo modo que se reconstruye la materialidad del robot, a partir del ensamblaje de relaciones sociales de las que participa (Law y Mol, 1995).

Integrando la ética de los cuidados para reflexionar y estudiar los efectos sociales y éticos vinculados a la robótica, el objeto de discusión ya no es el robot, el artefacto, sino la red de relaciones en las que ese robot participa (Vallès-Peris y Domènech, 2020b). Esta aproximación supera la conceptualización de las relaciones de los humanos con los robots desde un modelo binario, la conocida en inglés como Human-Robot Interaction (HRI). Según el paradigma HRI las relaciones de los humanos con los robots se sustentan en la idea de dos entidades independientes que interaccionan entre ellas.
Desde la ética de los cuidados, sin embargo, esta idea no tiene mucho sentido, y nos acercamos a los robots desde su integración en redes de relaciones de cuidados, una relación que podría llamarse algo así como un Robot Embedded in a Network (REN).

Desde un paradigma REN, por ejemplo, puede analizarse de qué manera la introducción de determinados artefactos robóticos puede cambiar la relación del hospital con el paciente, o de qué manera se transforman las competencias diagnósticas o de tratamiento del personal sanitario. En este sentido es relevante tener en cuenta, por ejemplo, algunos estudios sobre telemedicina, tecnologías a menudo integradas en los robots de cuidado. Estos estudios alertan de que este tipo soluciones tecnológicas encogen y parcelan al paciente, al mismo tiempo que dividen las habilidades clínicas del personal médico (Mort, May y Williams, 2003).

Una aproximación REN a los robots de cuidados también permite analizar de qué manera interaccionan los diversos agentes que participan en las relaciones de cuidados, en una situación particular. Por ejemplo, cuando una enfermera baña una persona con movilidad reducida con la ayuda de un robot, que la ayuda en la sujeción y limpieza del paciente, la facilidad de ejecución de la tarea que el robot incorpora no puede ir en detrimento de la relación visual y corporal que garantiza la buena relación de cuidados en ese momento (Mol, Moser y Pols, 2015). Desde el estudio del ensamblaje pueden identificarse cuáles son las variables o criterios relevantes para facilitar el 'buen cuidar' y cuál es la mejor manera posible de introducir estos artefactos para perseguir tal fin.

\subsection{Las prácticas cotidianas de cuidados con robots}

Cuando la reflexión se focaliza solamente en un desarrollo utópico o distópico de la robótica, algunos debates se sobredimensionan; mientras que la identificación de contextos, situaciones y controversias alrededor de artefactos que no responden a ese imaginario futuro, pasan desapercibidos. La 'simplicidad' aparente de algunos artefactos, que no integran las funcionalidades y capacidades complejas que se persiguen desde la prospectiva de robots androides altamente inteligentes, dificulta la identificación de problemas y la generación de un debate ético alrededor de algunos robots que ya se están utilizando. Desde la centralidad de las relaciones que supone una perspectiva REN, las implicaciones de introducir robots se sitúan en cada contexto de aplicación, y tienen que ver con un artefacto concreto en un entorno particular (Feng y Feenberg, 2008). El entramado constituye un marco de discusión singular y es en ese marco de prácticas cotidianas, particulares y específicas de cuidados donde emergen los problemas y las normas para resolver esos problemas.

En los entornos sanitarios y la literatura académica se considera que los robots de cuidados son aquellos que se utilizan en intervenciones terapéuticas específicas, por ejemplo, con niños y niñas autistas (Coeckelbergh et al., 2016) o en procesos de rehabilitación (Broekens, Heerink, y Rosendal, 2009), para reducir el dolor y la 
ansiedad antes de una intervención (Belpaeme et al., 2013), para hacer más llevadera la estadía en el hospital (Díaz-Boladeras et al., 2016), para ayudar a comer a personas con severas dificultades de movilidad (Nickelsen, 2018), como compañía de personas mayores que viven solas (Odekerken-Schröder, et al., 2020) o para monitorizar y dar apoyo en casos de demencia (Ienca, et al., 2016).

Es al menos curioso que algunos de los robots que más se han desarrollado e introducido a nivel global en los hospitales durante la COVID-19, los robots de desinfección, no se consideran robots de cuidados. Estos robots están diseñados para realizar tareas de desinfección, esterilización y facilitar cuarentenas, reduciendo las interacciones físicas entre personas (Khan, Siddique y Lee, 2020). Dichos artefactos utilizan radiaciones ultra-violeta, que ajustan según la detección de microbios, e integran sensores de movimiento para no irradiar a humanos. Podría considerarse que estos robots realizan actividades relacionadas con los cuidados, ya que todo aquello que tiene que ver con la limpieza se considera tradicionalmente como una actividad de cuidados, y está claramente recogido en las definiciones de Fisher y Tronto (1990) o van Wynsberghe (2015). Es interesante también el diseño de la nomenclatura a su alrededor: se habla de robots de desinfección, pero de mujeres de limpieza. Sin embargo, las implicaciones y efectos de introducir este tipo de artefactos no están presentes en las discusiones acerca de las consideraciones éticas y sociales de los robots de cuidados.

La omisión de este tipo de artefactos tiene relevantes consecuencias en cómo se articula el debate acerca de la robótica y la IA en los cuidados de la salud, invisibilizando determinados contextos y relaciones de cuidados, así como también eliminando la voz de las personas que participan en esas relaciones. ¿Qué sucede, por ejemplo, con las competencias tecnológicas de las mujeres de la limpieza de los hospitales? ¿De qué manera sus condiciones laborales y su salud pueden verse perjudicadas o beneficiadas con la introducción de estos artefactos? ¿Qué formas de relación e interacción prioriza el hospital con los pacientes de COVID-19?, y ¿cómo estas relaciones intervienen en el proceso de recuperación y salud de los enfermos? Este tipo de consideraciones son particularmente relevantes desde la ética de los cuidados, y posibilitan introducir en el ámbito de la robótica y la IA reflexiones sobre la fragmentación y organización jerárquica de los cuidados, su valoración y reconocimiento social, o las formas de desigualdad y discriminación que estas dinámicas conllevan (Vallès-Peris y Domènech, 2020a).

\subsection{La transformación de los entramados}

Los diversos debates éticos alrededor de las tecnologías de la salud tienen la capacidad de movilizar los imaginarios sociales, imaginarios que forman parte del entramado en el que se introduce el robot. La ética de los cuidados problematiza el enfoque deontológico según el cual los estándares éticos solo pueden llevarse a cabo mediante la declaración de principios, ya que estos principios describen aspiraciones morales, pero no posibilitan pensar de manera crítica ni desarrollar prácticas transformadoras (Tronto y Kohlen, 2018). El imaginario que acompaña la robótica desde su conceptualización como REN, requiere de cambios institucionales en el entramado en el que se organizan, gestionan y practican los cuidados con robots. Pueden agruparse éstas 'prácticas éticas' en tres cuestiones:

- La creación de sistemas locales de salud pública para el seguimiento del diseño e introducción de las tecnologías robóticas y de IA, aplicables también a los proyectos piloto o experimentales. Si las relaciones asistenciales y las prácticas de cuidado se sitúan en el centro del debate, es necesario establecer mecanismos que garanticen que la introducción de estos dispositivos responde a valores y prioridades médicas y asistenciales y no, por ejemplo, a intereses comerciales.

- La organización de foros participativos con aquellas personas implicadas en las relaciones de cuidados con robots, para debatir sobre sus miedos y esperanzas. Esto supone que la realización de procesos participativos con personas que diseñan y programan dispositivos robóticos y de IA, profesionales de la medicina, cuidadoras formales e informales, pacientes y familiares, debería ser una práctica habitual. A través de estos procesos se podrán identificar problemas y preocupaciones cotidianas, así como integrar diferentes puntos de vista y necesidades.

- La elección de pequeñas acciones progresivas para 'mantener bajo control' los efectos o consecuencias de su implementación. Pequeñas acciones basadas en bucles de retroalimentación progresivos con sistemas de monitorización y debate constante. Estos bucles de retroalimentación garantizarán que la introducción de dispositivos robóticos y de IA estén en consonancia con las prácticas de cuidados en un hospital o entorno sanitario concretos, e integre las preocupaciones de los diferentes actores implicados.

\section{Conclusiones}

En este artículo he explorado cómo podemos repensar la robótica y la IA en el ámbito de los cuidados de la salud, desde la ética de los cuidados. La propuesta que he desarrollado parte de la idea de continuum entre cuidados, política, ética y tecnología. Este planteamiento se articula alrededor de lo que he llamado un paradigma REN (de sus siglas en inglés Robot Embedded in a Network, un robot integrado en un entramado), una manera de acercarse a las relaciones con los robots desde su dimensión ético-política. Esta propuesta asume que el centro del debate es el entramado de relaciones de cuidados de las que participa el robot, relaciones desiguales que, a la vez, requiere de transformaciones institucionales y de la participación de diversos actores, para garantizar una robótica e IA responsables y al servicio del bien común. 
A pesar de que en este artículo he argumentado teóricamente una manera de repensar la robótica y la IA desde la ética de los cuidados, el desarrollo de esta propuesta requiere del acompañamiento y simbiosis con el trabajo empírico. Según la propia lógica de la ética de los cuidados, localizada en contextos y situaciones particulares, explorar las controversias y los debates éticos alrededor de la robótica requiere de un análisis situado sobre cómo se (co)conforman las relaciones entre los diversos nodos del entramado de los que participa el robot.
Desde este planteamiento un futuro utópico con robots de cuidados es un futuro en el que el que el 'Obuen cuidar' sea el centro del debate. Si esta es la utopía, en el presente de nuestras cotidianidades, entonces, la ética de los cuidados es el espacio privilegiado para combatir utopías depredadoras con la vida, el entorno y las relaciones. Una ética-política que acompañe imaginarios y prácticas transformadoras con las tecnologías, para vivir en nuestro mundo común de la mejor manera posible.

\section{Referencias}

Agrela Romero, B., Martín Palomo, M. T. M. y Langa Rosado, D. (2010). Modelos de provisión de cuidados: Género, familias y migraciones. Nuevos retos y configuraciones para las políticas públicas. Alternativas, 17, 9-17. https://doi.org/10.14198/ ALTERN2010.17.1

Belpaeme, T., Baxter, P., Greeff, J. De, Kennedy, J., Looije, R., Neerincx, M., ... Coti, M. (2013). Child-robot interaction: perspectives and challenges. En Lecture Notes in Artificial Intelligence (R. Goebel, Y. Tanaka, W. Wahlster, pp. 452-459). Berlin: Springer-Verlag Berlin.

Bijker, W. E. (2009). How is technology made?-That is the question! Cambridge Journal of Economics, 34(1), 63-76. https://doi. org/10.1093/cje/bep068

Block, F. y Somers, M. (1984). Beyond the economistic fallacy: the holistic social science of Karl Polanyi. En Vision and Method in Historical Sociology (T. Skocpol, pp. 47-84). Nueva York: Cambridge University Press.

Braidotti, R. (2000). Sujetos nómades. Corporización y diferencia sexual en la teoría feminista contemporánea. Buenos Aires: Paidós.

Breazeal, C. (2011). Social robots for health applications. 2011 Annual International Conference of the IEEE Engineering in Medicine and Biology Society, 5368-5371. https://doi.org/10.1109/IEMBS.2011.6091328

Broekens, J., Heerink, M. y Rosendal, H. (2009). Assistive social robots in elderly care: a review. Gerontechnology, 8(2), 94-103. https://doi.org/10.4017/gt.2009.08.02.002.00

Callon, M. (1998). El proceso de construcción de la sociedad. El estudio de la tecnología como herramienta para el análisis sociológico. En Sociología simétrica. Ensayos sobre ciencia, tecnología y sociedad (M. Domènech y F. J. Tirado, pp. 143170). Barcelona: Gedisa.

Calo, R. (2017). Artificial Intelligence Policy: A Roadmap. SSRN Electronic Journal, 1-28. https://doi.org/10.2139/ssrn.3015350

Carrasco, C. (2019). El trabajo de cuidados: historia, teoría y políticas. Madrid: Los libros de la Catarata.

Carretero Pasín, A. E. (2005). Imaginario y utopías. Athenea Digital. Revista de Pensamiento e Investigación Social, 1(7), 40. https://doi.org/10.5565/rev/athenead/v1n7.180

Castoriadis, C. (1975). La institución imaginaria de la sociedad. Vol 2. El imaginario social y la institución. Barcelona: Tusquets Editores S.A.

Castoriadis, C. (2005). Una sociedad a la deriva. Entrevistas y debates (1974-1997). Buenos Aires: Katz Editores.

Coeckelbergh, M. (2012). Hacking Feenberg. Symploke, 20(1), 327-330.

Coeckelbergh, M., Pop, C., Simut, R., Peca, A., Pintea, S., David, D. y Vanderborght, B. (2016). A survey of expectations about the role of robots in robot-assisted therapy for children with asd: ethical acceptability, trust, sociability, appearance, and attachment. Science and Engineering Ethics, 22(1), 47-65. https://doi.org/10.1007/s11948-015-9649-x

Díaz-Boladeras, M., Angulo, C., Domènech, M., Albo-Canals, J., Serrallonga, N., Raya, C. y Barco, A. (2016). Assessing pediatrics patients' psychological states from biomedical signals in a cloud of social robots. In XIV Mediterranean Conference on Medical and Biological Engineering and Computing, 57, 1179-1184. https://doi.org/10.1007/978-3-319-32703-7_229

Domínguez Alcón, C. (2018). Transformación del cuidado: Retos y responsabilidades. Temperamentvm: Revista Internacional de Historia y Pensamiento Enfermero, 14, e12287.

Duclos, V. y Criado, T. S. (2020). Care in Trouble: Ecologies of Support from Below and Beyond. Medical Anthropology Quarterly, 34(2), 153-173. https://doi.org/10.1111/maq.12540

Dupuy, J.-P. (1991). El pánico. Barcelona: Gedisa Editorial.

Feenberg, A. (2010). Between Reason and Experience. Essays in Technology and Modernity. Cambridge, MA y Londres: The MIT Press.

Feng, P. y Feenberg, A. (2008). Thinking about design: critical theory of technology and the design process. En Philosopy and Design. From Engineering to Architecture (P.E. Vermaas, P. Kroes, A. Light, S. Moore, 105-118). Springer Science+Business Media B.V.

Fisher, B. y Tronto, J. (1990). Toward a feminist theory fo caring. En Circles of Care: Work and Identity in Women's Lives (K. Abel y M. K. Nelson, pp. 35-62). Albany: SUNY Press.

FRA, (2020). Getting the Future Right. Artificial Intelligence and Fundamental Rights. Luxembourg: Publications Office of the European Union.

Grunwald, A. (2010). From speculative nanoethics to explorative philosophy of nanotechnology. NanoEthics, 4(2), 91-101. https://doi.org/10.1007/s11569-010-0088-5

Hagendorff, T. (2020). The ethics of AI ethics: an evaluation of guidelines. Minds and Machines, 30(1), 99-120. https://doi. org/10.1007/s11023-020-09517-8 
Ienca, M., Jotterand, F., Vica, C. y Elger, B. (2016). Social and assistive robotics in dementia care: ethical recommendations for research and practice. International Journal of Social Robotics, 8(4), 565-573. https://doi.org/10.1007/s12369-016-0366-7

International Federation of Robotics (IFR). (2018). World Robotics/Statistics \& Resources. Recuperado de: https://ifr.org

Khan, Z. H., Siddique, A. y Lee, C. W. (2020). Robotics utilization for healthcare digitization in global COVID-19 Management. International Journal of Environmental Research and Public Health, 17(11), 3819. https://doi.org/10.3390/ijerph17113819

Kritikos, M.(2020). Ten technologies to fight coronavirus. Brussels: European Union. Recuperado de: https://doi.org/10.2861/58070

Law, J. y Mol, A. (1995). Notes on Materiality and Sociality. The Sociological Review, 43(2), 274-294. https://doi. org/10.1111\%2Fj.1467-954X.1995.tb00604.x

López Gómez, D. (2015). Little arrangements that matter. Rethinking autonomy-enabling innovations for later life. Technological Forecasting and Social Change, 93, 91-101. https://doi.org/10.1016/j.techfore.2014.02.015

Martínez, L. M., Araiza, A., Garay, A. I. y Peñaranda, M. del C. (2011). Género, espacio doméstico y socialización del cuidado: Algunas perspectivas de cambio. International Journal of Developmental and Educational Psychology, 5(1), $181-186$.

Matsuzaki, H. y Lindemann, G. (2016). The autonomy-safety-paradox of service robotics in Europe and Japan: a comparative analysis. AI and Society, 31(4), 501-517. https://doi.org/10.1007/s00146-015-0630-7

Mejia, C. y Kajikawa, Y. (2017). Bibliometric analysis of social robotics research: identifying research trends and knowledgebase. Applied Sciences, 7(12), 1316. https://doi.org/10.3390/app7121316

Mol, A., Moser, I. y Pols, J. (2015). Care in practice: On tinkering in clinics, homes and farms. Bielefield: Verlag.

Mort, M., May, C. R. y Williams, T. (2003). Remote doctors and absent patients: Acting at a distance in telemedicine? Science Technology and Human Values, 28(2), 274-295. https://doi.org/10.1177/0162243902250907

Moyà-Köhler, J. (2018). Sincronitzant autonomies: estudi d'un servei de vida independent per a persones amb la síndrome de Down (Tesis Doctoral, Universitat Autònoma de Barcelona). Recuperado de: https://www.tdx.cat/handle/10803/666756\#page=1

Muller, V. C. (2020). Ethics of Artificial Intelligence and Robotics (Stanford Encyclopedia of Philosophy). Stanford Encyclopedia of Philosophy, 1-30. Recuperado de: https://plato.stanford.edu/entries/ethics-ai/

Nickelsen, N. C. M. (2018). Feeding assistive robotics, socio-technological imaginaries and care: the case of Bestic. En REELER Working Paper Series. Copenhagen: Aarhus University.

Nordmann, A. y Rip, A. (2009). Mind the gap revisited. Nature Nanotechnology, 4, 273-274. https://doi.org/10.1053/j. seminoncol.2017.07.003

Odekerken-Schröder, G., Mele, C., Russo-Spena, T., Mahr, D. y Ruggiero, A. (2020). Mitigating loneliness with companion robots in the COVID-19 pandemic and beyond: an integrative framework and research agenda. Journal of Service Management, 31(6), 1149-1162. https://doi.org/10.1108/JOSM-05-2020-0148

Pistono, F. y Yampolskiy, R. V. (2016). Unethical Research: How to Create a Malevolent Artificial Intelligence. ArXiv, 1-6.

Polany, K. (2015). La Gran Transformación. Crítica del liberalismo económico. Barcelona: Virus editorial.

Precarias a la deriva (2004). A la deriva por los circuitos de la precariedad femenina: precarias a la deriva. Madrid: Traficantes de Sueños.

Puig de la Bellacasa, M. (2011). Matters of care in technoscience: assembling neglected things. Social Studies of Science, 41(1), 85-106. https://doi.org/10.1177/0306312710380301

Puig de la Bellacasa, M. (2017). Matters of Care. Speculative Ethics in More Than Human Worlds. Minneapolis: University of Minnesota Press.

Russell, S. J. y Norvig, P. (2003). Artificial Intelligence: A Modern Approach. New Jersey: Prentice Hall Series in Artificial Intelligence.

Šabanović, S. (2010). Robots in Society, Society in Robots. International Journal of Social Robotics, 2(4), 439-450. https://doi. org/10.1007/s12369-010-0066-7

Sánchez Criado, T. y Rodríguez Giralt, I. (2017). Caring through design? En torno alla silla and the'joint problem-making'of technical aids. En Care and Design: Bodies, Buildings, Cities (C. Bates, R. Imrie, y K. Kullman, 198-218). Wiley Blackwell.

Schaal, S. (2007). The new robotics-towards human $\square$ centered machines. HFSP Journal, 1(2), 115-126. https://doi. org/10.2976/1.2748612

Serres, M. (2013). Pulgarcita. Buenos Aires: Fondo de Cultura Económica.

Sharkey, N. (2008). Computer Science. The Ethical Frontiers of Robotics. Science, 322(5909), 1800-1801. https://doi.org/10.1126/ science. 1164582

Sharkey, N. y Sharkey, A. (2012). The eldercare factory. Gerontology, 58(3), 282-288. https://doi.org/10.1159/000329483

Sparrow, R. y Sparrow, L. (2006). In the hands of machines? The future of aged care. Minds and Machines, 16(2), $141-161$. https://doi.org/10.1007/s11023-006-9030-6

Steels, L. y Mantaras, R. L. De. (2018). The Barcelona declaration for the proper development and usage of artificial intelligence in Europe. AI Communications, 31, 485-494. https://doi.org/10.3233/AIC-180607

Tronto, J. (2018). La democracia del cuidado como antídoto frente al neoliberalismo. En El futuro del cuidado. Comprensión de la ética del cuidado y práctica enfermera (C. Domínguez Alcón, H. Kohlen, y J. Tronto, 7-19). Barcelona: Ediciones San Juan de Dios.

Tronto, J. y Kohlen, H. (2018). ¿Puede ser codificada la ética del cuidado? En El futuro del cuidado. Comprensión de la ética del cuidado y práctica enfermera (C. Domínguez Alcón, H. Kohlen, y J. Tronto, 20-32). Barcelona: Ediciones San Juan de Dios.

Vallès-Peris, N., Argudo-Portal, V. y Domènech, M. (en prensa). Manufacturing Life, What Life? Ethical Debates around Biobanks \& Social Robots. NanoEthics.

Vallès-Peris, N. y Domènech, M. (2020a). Roboticists' imaginaries of robots for care: the radical imaginary as a tool for an ethical discussion. Engineering Studies, 12(3), 157-176. https://doi.org/10.1080/19378629.2020.1821695

Vallès-Peris, N. y Domènech, M. (2020b). Robots para los cuidados. La ética de la acción mesurada frente a la incertidumbre. Cuadernos de Bioética, 31(101), 87-100. https://doi.org/10.30444/CB.54

van der Plas, A., Smits, M. y Wehrmann, C. (2010). Beyond speculative robot ethics: a vision assessment study on the future of the robotic caretaker. Accountability in Research, 17(6), 299-315. https://doi.org/10.1080/08989621.2010.524078 
van Wynsberghe, A. (2013). Designing Robots for Care: Care Centered Value-Sensitive Design. Science and Engineering Ethics, 19(2), 407-433. https://doi.org/10.1007/s11948-011-9343-6

Van Wynsberghe, A. (2015). Healthcare Robots. Ethics, Design and Implementation. Londres y Nueva York: Routledge.

Verbeek, P.-P. (2008). Morality in Design. Design Ethics and the Morality of Technological Artifacts. En Philosophy and Design (P. E. Vermaas, 91-103). Dordrecht: Springer.

Veruggio, G. (2005). The Birth of Roboethics. En IEEE International Conference on Robotics and Automation, Workshop on Roboethics. Recuperado de: https://philpapers.org/archive/VERTBO-3.pdf

Willems, D. y Pols, J. (2010). Goodness! The empirical turn in health care ethics. Medische Antropologie, 22(1), 161-170.

Winance, M. (2006). Trying Out the Wheelchair: The Mutual Shaping of People and Devices through Adjustment. Science, Technology \& Human Values, 31(1), 52-72. https://doi.org/10.1177/0162243905280023 